\title{
A preliminary phylogeny of cave trechine beetles from China (Coleoptera: Carabidae: Trechini)
}

\author{
Sunbin Huang ${ }^{\ddagger}$, Mingyi Tian§, Arnaud Faillel \\ ‡ MECADEV, UMR 7179 CNRS-MNHN, Muséum National d'Histoire Naturelle, Paris, France \\ $\S$ Department of Entomology, College of Agriculture, South China Agricultural University, Guangzhou, China \\ | State Museum of Natural History Stuttgart, Stuttgart, Germany
}

Corresponding author: Mingyi Tian (ㅆtian@scau.edu.cn), Arnaud Faille (faille@mnhn.fr)

Received: 09 Mar 2020 | Published: 16 Mar 2020

Citation: Huang S, Tian M, Faille A (2020) A preliminary phylogeny of cave trechine beetles from China

(Coleoptera: Carabidae: Trechini). ARPHA Conference Abstracts 3: e51897.

https://doi.org/10.3897/aca.3.e51897

\begin{abstract}
Coleoptera is one of the most successful groups among the subterranean fauna. Within Carabidae, 25 tribes have been reported in subterranean habitats, including the first representative of the tribe Patrobini, just described from Southern China. Amongst them, Trechini is the most diverse and cave-specialized group, and the numerous lineages of this group which have diversified underground make it an excellent model for the study of evolutionary mechanisms.

In China, 152 species within 52 genera of Trechini have been described so far. In order to unveil the relationships and origin of this remarkable biodiversity and to understand the evolutionary success of this group, we infer the first phylogeny of the group by using molecular sequence data from four genes (two mitochondrial markers: cox1 and 16S; plus two nuclear: $18 \mathrm{~S}$ and $28 \mathrm{~S}$ ). We found that the Chinese cave Trechini do not form a monophyletic clade and we identified three main independent subterranean clades. To define the systematic position of the main clades on a worldwide scale, we gathered other Trechini sequence data from different lineages and part of the world. The preliminary results will be presented in this talk.
\end{abstract}




\section{Keywords}

aphaenopsian, cavernicolous, ground beetles, molecular

\section{Presenting author}

Sunbin Huang

\section{Presented at}

The 25th International Conference on Subterranean Biology 